\title{
Dynamic location and forwarding pointers for mobility management
}

\author{
Charles Abondo and Samuel Pierre* \\ Mobile Computing and Networking Research Laboratory (LARIM), Canada
}

\begin{abstract}
GSM and IS-41 are two mobility management standards widely used in second generation networks. These two standards lean on a centralized architecture made up of home location registers (HLRs) and visitor location registers (VLRs). From these standards, the location update and search procedures always imply interrogation of the HLR, even if the two mobile terminals that want to communicate are in the same location area. Given the limited bandwidth of the radio operator channel and the new time sensitive applications of third-generation systems, such an approach of mobility management is not convenient for the next generation mobile networks. This paper proposes a method for reducing the processing load and the signalization traffic generated by update and search location procedures compared to IS-41 standard. Taking into account the specific characteristics of the traffic in the mobile networks, it introduces a semi-dynamic approach based on a hybrid architecture using forwarding pointers without the load related to the dynamic models. Numerical results show that such a method significantly improves the efficiency of location procedures.
\end{abstract}

Keywords: Mobility management, 3G mobile networks, dynamic location, wireless systems, location update, location search

\section{Introduction}

In traditional wired networks like telephone networks, there is a fixed relation between a terminal and its location. The change of terminal location generally implies a network management that can be carried out with difficulty by the user. The calls towards a given terminal are always forwarded to the location of this one because there is no distinction between a terminal and its location. On the contrary, mobile systems support users who are free to move in space and time. This capability to move is called roaming. Thus, the user's network access point changes when he/she moves and the identity of the mobile unit does not implicitly provide its location. Therefore, we need a mechanism to locate the user under the network's area. This mechanism is named roaming management or mobility management.

The current location management techniques consist of dividing the coverage area of the system into several location areas (LAs). Consequently, as soon as a unit enters a new location area, it must bring back to the network its new location server, according to a procedure known as location update. In the same way, to communicate with a mobile unit (MU), the first operation consists of determining the current position of this unit, according to a procedure known as location search [2,3].

There are two mobility management standards in second generation networks, GSM and IS-41. These two standards lean on a centralized architecture made up of home location registers (HLRs) and visitor

\footnotetext{
${ }^{*}$ Corresponding author: Samuel Pierre, Department of Computer Engineering, Ecole Polytechnique of Montreal, P.O. Box 6079, Station Centre-ville, Montréal, Que., Canada H3C 3A7. Tel.: +1 5143404711 ext. 4685; Fax: +1 514 340 3240; E-mail: samuel.pierre@polymtl.ca.
} 


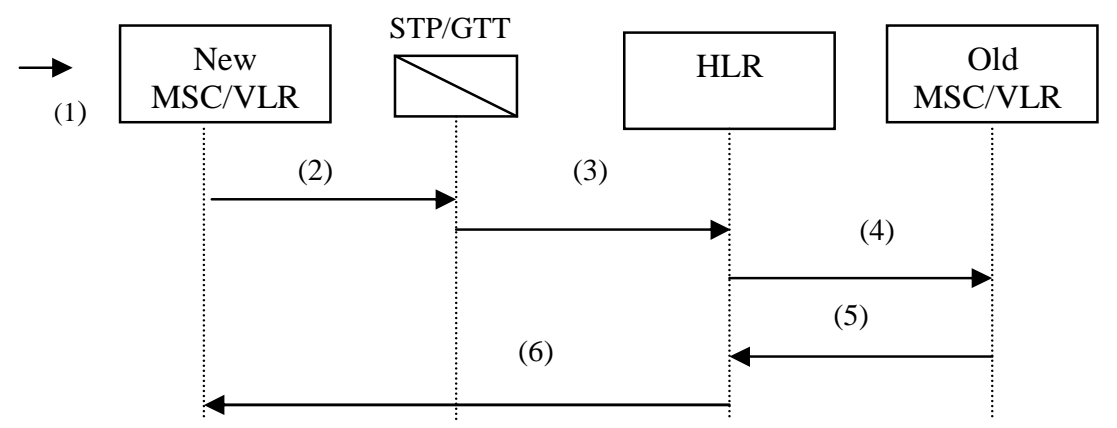

Fig. 1. Updating procedure according to IS-41 standard.

location registers (VLRs). From these standards, the location update and search procedures always imply interrogation of the HLR, even if the two mobile terminals that want to communicate are in the same location area. The traffic generated by the mobility management in GSM system currently represents up to $80 \%$ of the access channel and can reach values ten times higher than those of the incoming and outgoing calls. This is not a major problem for the current networks, given the weak mobility and penetration rate of the existing mobile systems. On the other hand, third-generation systems suppose four impact levels on mobility management:

- Space Level: mobility passed from local to regional and finally to international (global roaming);

- User's density Level: quasi-exponential progression of the number of users;

- Level of the traffic by subscriber: increased use of mobiles;

- Level of the services offered: voice, Internet, multimedia, mobile-commerce.

Given the limited bandwidth of the radio operator channel and the new time sensitive applications of third-generation networks, it is imperative to reduce the signalization and processing load generated by the current mobility management methods.

This paper proposes a new mechanism to reduce the processing load and the signalization traffic generated by update and search location procedures compared toIS-41 standard. Section 2 summarizes IS-41 standard and various mobility management methods listed in the literature. Section 3 explains the proposed mobility management model. Section 4 presents the numerical results of the performance study of the proposed strategy compared to that of IS-41 standard. Section 5 outlines some concluding remarks.

\section{Some current methods of mobility management}

In this section, we first present the IS-41 mobility management such as defined in the revision C of IS-41 standard [2] and then various mobility management proposals found in the literature.

\subsection{IS-41 standard}

IS-41 standard defines two mobility management procedures: procedure known as location update and a procedure known as location search. Figure 1 illustrates the messages exchanged between the network and the mobile unit for a location update procedure. The operations are defined as follows:

1. When an MU enters a new LA, it sends a registration message to the MSC/VLR through the base station. 


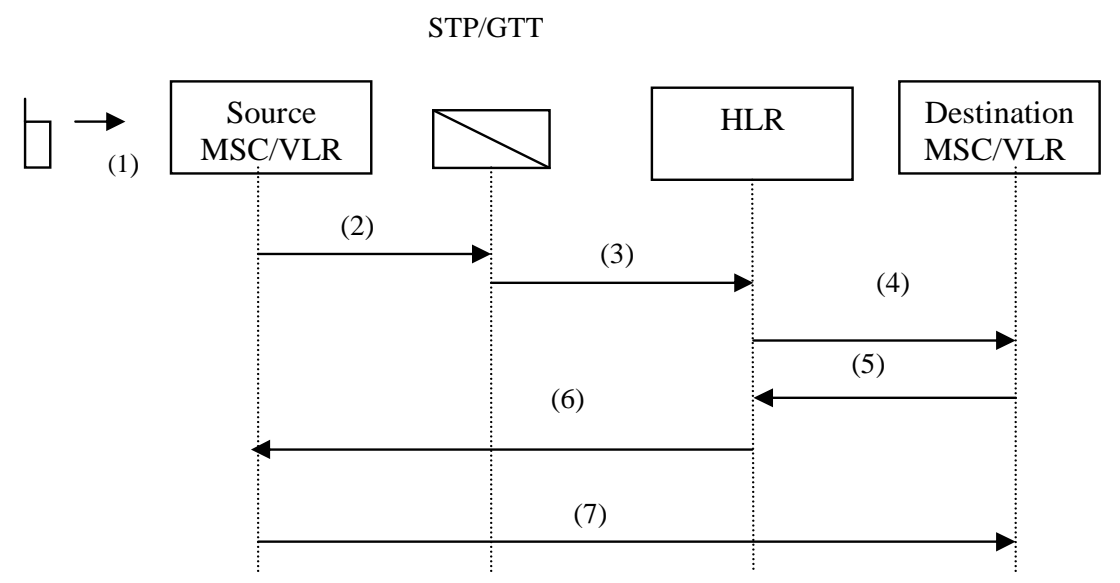

Fig. 2. Location search procedure according to IS-41 standard.

2. The MSC/VLR of the new LA registers the MU and sends a registration notification message to the HLR via the signal transfer point (STP).

3. Based on the MU's identification number, the STP execute the global title translation (GTT) procedure to determine the HLR of the MU. The corresponding message is then forwarded to the HLR.

4. The HLR sends a registration cancellation message to the previous VLR.

5. The VLR previously visited deletes the registration of the MU and sends a cancellation acknowledgement message (regcanc) to the HLR.

6. The HLR acknowledges the location update by sending an acknowledgement to the new MSC/VLR. The HLR provides the MU's profile in this message. After receiving this message, the MSC/VLR starts providing service to the MU.

In a similar way, to deliver an incoming call, the network must access the databases in order to determine in which location area is the MU. Figure 2 illustrates the search location procedure of IS-41 standard. The operations are defined as follows:

1. An incoming call from a mobile unit or from a fixed terminal is received by the source MSC/VLR.

2. The source MSC/VLR sends a location request message to the HLR of the called MU via the STP.

3. The STP executes the GTT procedure to determine the HLR the message must be forwarded to.

4. Upon receipt of the message, the HLR determines the MSC/VLR of the called MU and sends a routing request message to the MSC/VLR destination.

5. The MSC/VLR destination assigns a temporary location directory number (TLDN) for the call which is sent to the HLR.

6. The HLR relays the TLDN to the source MSC/VLR.

7. The source MSC/VLR routes the call to the destination MSC/VLR.

These two procedures generate a strong signalization load and a heavy database access traffic especially when the mobile unit is located far from the HLR. For example, a mobile unit being in Vancouver and its HLR being in Montreal sends update messages in Montreal even when it moves in Vancouver area. In the same way, when the mobile unit receives calls from the same location area than him/her, the system sends location request messages to the HLR in Montreal. 


\subsection{Related work}

Many work was devoted to the impact of roaming management in the mobile networks [7-9,11]. They have all as a common objective to reduce the traffic load generated by the mobility management.

A strategy based on pointers according to the moves of the MUs is proposed in [6]. The main idea of this proposal is to create a pointer of the old location area towards the new one instead of making an update each time the mobile unit moves to a new location area. When there is an incoming call, the network determines the position of the mobile unit at the beginning of the chain of pointers and follows the chain from the old LA to the current one. To minimize the time in the search of a mobile unit, the length of chain is limited to a given value $\mathrm{K}$. If the length of chain reached the value $\mathrm{K}$, the next location update is reported to the HLR. The authors have demonstrated that according to the user's mobility and the number of calls, this procedure does not always improve the location update and search procedures. In this case, it is necessary to determine under which conditions this procedure is better than IS-41 standard.

Another strategy of anchor LA per user is introduced in [5] and reduces the cost location management by eliminating the carry forward from the location to the HLR. Each mobile unit is seen assigning a VLR as being its local database. Instead of transmitting the updates to the HLR, those are transmitted to the local database of the mobile. Since the local database is close to the mobile, the signalization traffic is thus reduced. The local database or anchor LA keeps a pointer to the current LA. At the time of an incoming call, the HLR requests the local database of the mobile which forwards the call to the current VLR. There are two ways of determining the local database of each mobile: the static local database and the dynamic local database. In the first case, the local database is the VLR where it received its last call. This method completely eliminates the updates on the level from the HLR but does not always minimize the cost of location. In the second case, the local database is the VLR where it received its last call but this time the network determines if the local database must be moved dependently on the mobility parameters of the user. It is shown that this method always results in a cost less than or equal to IS-41 standard.

In [4], an intermediate level database, called DR (Directory register), is introduced, assuming that DR are localized on the level of the STP of the signalization network SS7 and that each DR covers several MSCs. DR stores and processes the information of the users under its area. It also contains three types of location pointers related to local or remote users and has the capacity to forward the location update and search messages of the mobile units moving in directory area (DA) to the network element indicated by the location pointers. The pointers are used in order to distribute information of the users over the network. Since the mobility pattern and the rate of calls vary from one user to another, the configuration of the pointers is given in a single way for each mobile. According to this model, DR periodically determines the information distribution location strategy for each mobile. The location cost is reduced if a distant pointer towards the called mobile unit exists. However, an additional load of processing is necessary for each move to update the pointers.

In [3], a distributed call processing structure is introduced to prevent call processing delays caused by congestion of the signaling load in call processing and registration. The proposed call processing procedure reuses previous information based on a cache updating using ATM multicasting to reduce the signaling load for the total network, for the connection establishment delay, and for the query frequency. The HLR accepts registration information, transmits the information to all caches, and requests renewal for the cache using the ATM multicasting function for location registration of the terminal. Then, if the corresponding information is present, each cache performs the renewal. Otherwise, the cache discards 


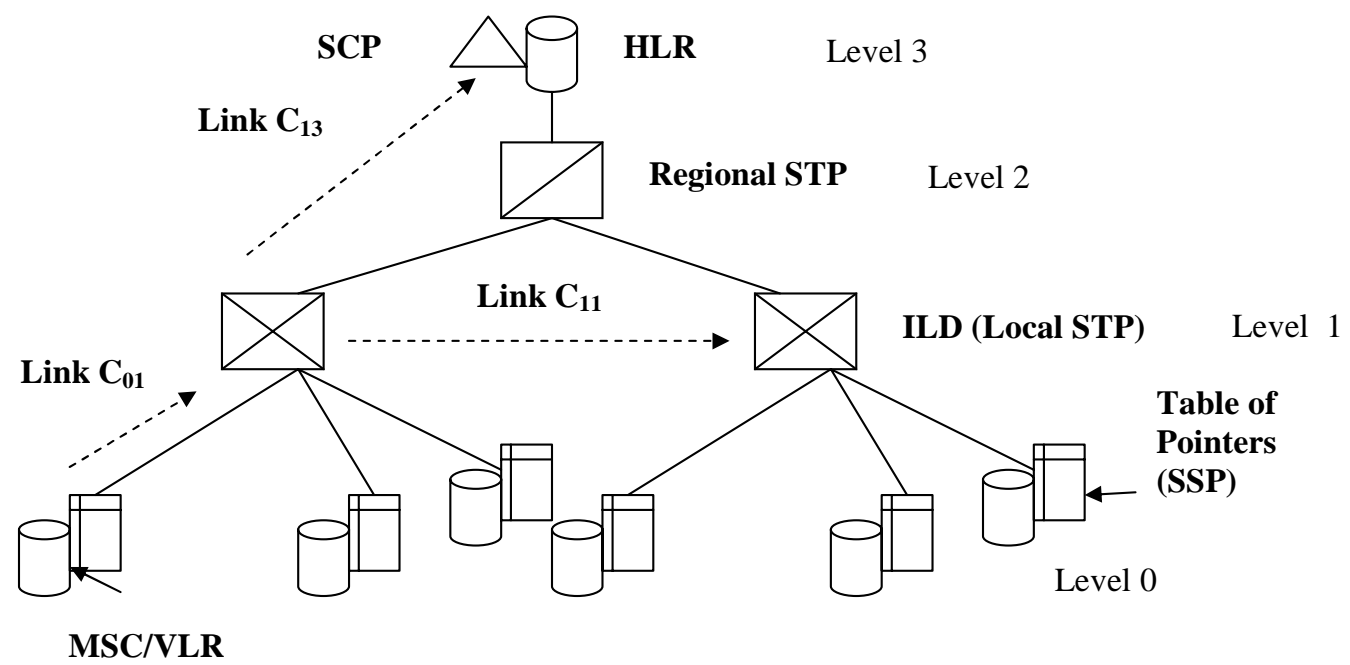

Fig. 3. Hybrid architechure.

the renewal request. The high hit ratio of cache reduces additional load by cache miss if the HLR and the cache always have the same information. Performance analysis shows that the call processing is considerably improved in the case of a high call mobility ratio (CMR).

In [10], a two-level pointer strategy is introduced to reduce the tracking delay in PCS systems. The first level of pointers is setup between some VLRs refer as Mobility Agents (MA). The second level of pointers is setup between LAs in the same Mobility Agent zone. Every time an MU moves from one LA to another, it is tracked using the first or the second level of pointers depending on the move. Performance analysis shows that the network signaling is reduced for users with low CMR.

We believe that the mobility management in third-generation systems will have to separate the optimization of location update and search procedures, to reduce updates and queries of the home location register (HLR), to integrate the users' profile in the mobility procedures, to distribute the users information in the network, to conceive a database architecture neither centralized nor distributed, and to release the constraints on the call mobility ratio (CMR). It should be noticed that this is not a major disadvantage because we think that the third-generation systems will be based on a multi-layer location area architecture. So there will be various classes of users depending on the mobility parameters of the latter. It will be thus possible to apply a type of algorithm to a class of user corresponding to an interval of the call mobility ratio (CMR).

\section{The proposed location management scheme}

The architecture proposed is based on the structure of signaling system number 7 (SS7) network with a new level of intermediate location database (ILD) and a table of pointers. The sites and the operations of the HLR, VLRs and MSCs remain practically unchanged. Figure 3 presents such an architecture by indicating all the basic elements involved in the mobility management. This architecture will be called hybrid architecture or hybrid algorithm. The times of propagation and transmission between the various layers or levels are represented by the costs of $C_{i j}$ links. 


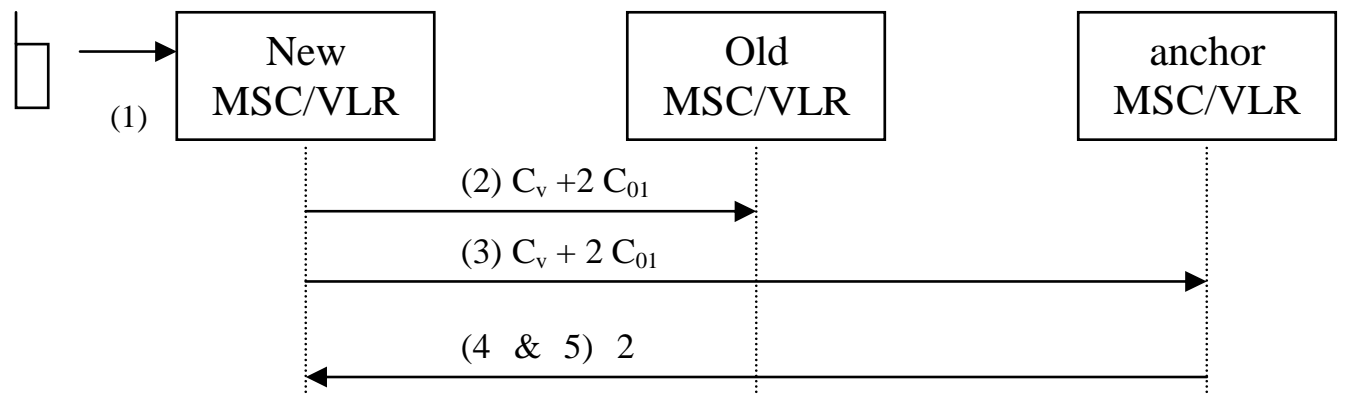

Fig. 4. Updating procedure for an intra-ILD move.

\subsection{Basic idea}

We suppose that ILDs are installed on the level of STPs of the SS7 network. Each ILD serves a certain number of MSCs and can route calls. The intermediate location databases contain users' profiles of all the MUs moving under their coverage area. They can, moreover, create a distant pointer towards other ILDs or towards a MSC/VLR from another ILD, in order to forward the requests towards the network elements. We assume that the system can know at any time the cost of any link. This allows optimizing the pointer cost. For example, when the cost of link $c_{11}$ between two ILDs is more expensive than the rise towards the HLR $c_{13}$, the requests will be transmitted according to the least expensive path.

The use of ILDs makes it possible to reduce the traffic load by minimizing the number of requests transmitted to HLR. For example, every time an MU moves inside an ILD, there is no update to the HLR. It also allows reducing the traffic load by decreasing the user's time of search because the major part of calls are local calls. Therefore, we do not need anymore to go up to the HLR to determine the location of the called MUs.

A table of pointers is also added to the level of each VLR covering a location area. This table contains a pointer towards the zone of current location of each mobile unit having this location area as anchor. It is necessary to distinguish the intra-ILD moves and the inter-ILD moves. An intra-ILD move occurs when the new and the old location area are anchor to the same ILD. An inter-ILD move occurs when the new and the old location area belong to two different ILDs. It should be noted that the HLR preserves the identity of the ILD under which move the MU and its anchor LA. We suppose that each mobile unit preserves the identity of its anchor LA. When an MU moves under the coverage area of an ILD, its anchor LA creates a pointer towards the new visited LA. The anchor strategy optimizes the updates during an intra-ILD move because the carry forward of move is done only on the MSC/VLR and not of the ILD. Thus, no update is necessary to the level of the HLR or the ILD. When a mobile unit moves towards a new ILD, the new location area becomes its new anchor LA and the old one is deleted.

\subsection{Location update and location search procedures}

When a user moves from one location area to another one or when that user receives a call, two procedures are carried out on the level of the network elements. We present in this section the various update and search algorithms.

\subsubsection{Location update procedure}

Figures 4 and 5 illustrate respectively the intra-ILD and inter-ILD update. The operations for an intra-ILD move are described as follows: 


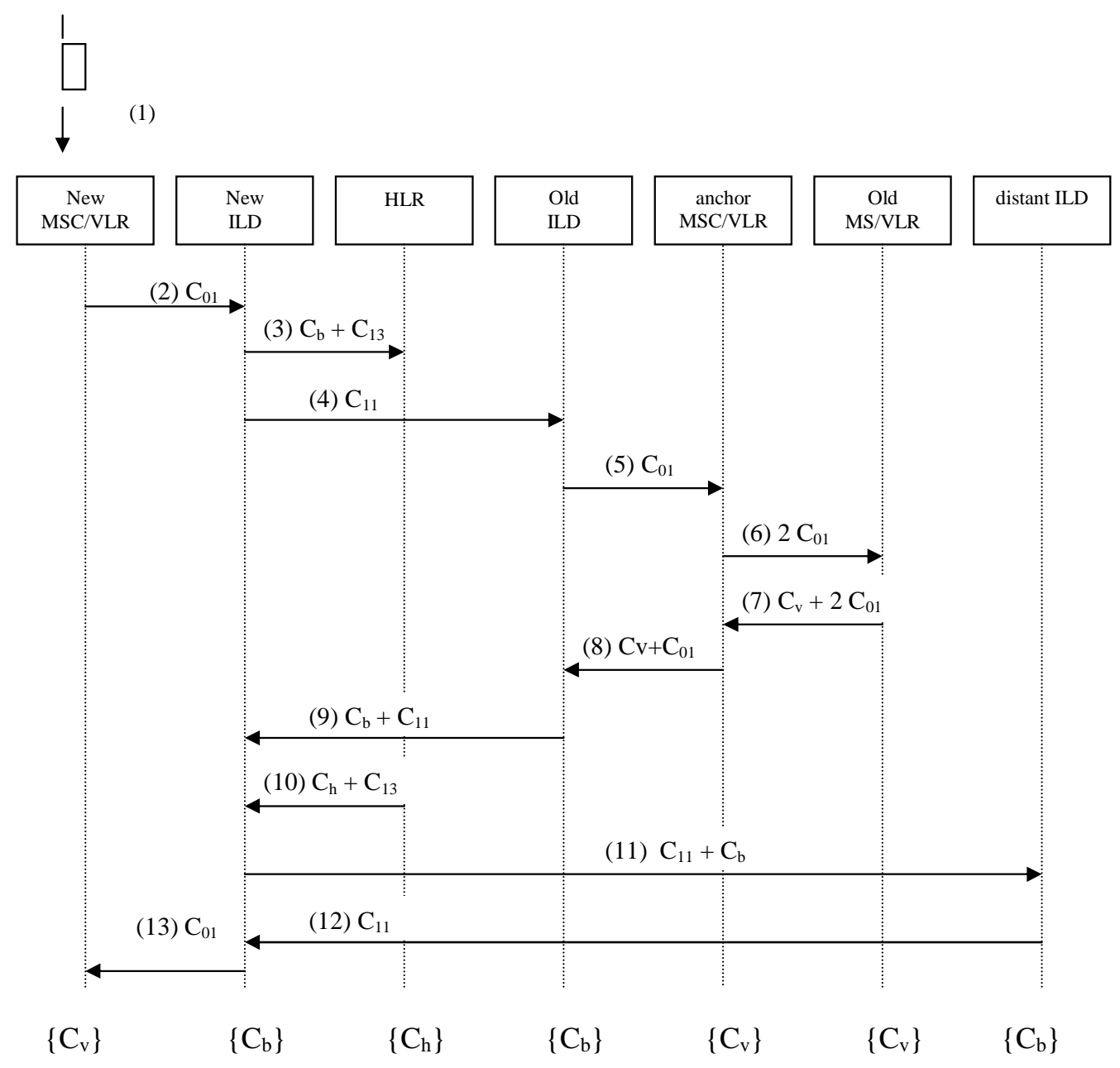

Fig. 5. Updating procedure for an inter-ILD move.

1. The MU moves to a new LA and sends a location update message to the MSC/VLR of the new LA.

2. The MSC of the new LA registers the MU and its anchor MSC/VLR, then sends a cancellation message to the old MSC.

3. The new LA also sends a message to the anchor MSC/VLR in order to create a pointer to its LA. Thus, no update is carried out on the level of the current ILD.

4. and 5. The new LA receives an acknowledgement from both the old and the anchor MSC/VLR.

When move is inter-ILD, one carries out the operations of Fig. 5 described as follows:

1. The MU moves to a new LA and sends a location update message to the MSC/VLR of the new LA.

2. The MSC of the new LA registers the MU and deduces that it is the new anchor MSC/VLR. It then sends a registration notification to the ILD.

3. and 4. The local ILD registers the MU and forwards the registration message to the HLR and to the old ILD.

4. The old ILD sends a cancellation message to the old anchor MSC/VLR. 


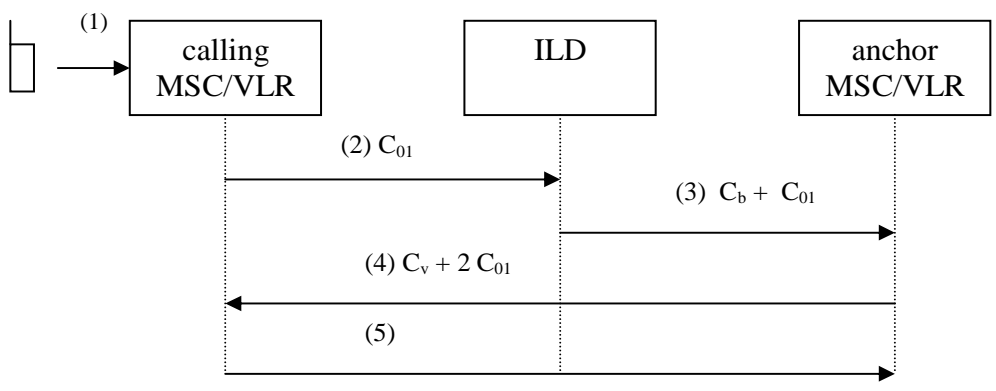

Fig. 6. Location search procedure (scenario 1).

5. The old anchor VLR sends a cancellation message to the previously visited LA.

6. The previously visited LA deletes the MU's profile and sends a cancellation acknowledgement to the anchor VLR.

7. The old anchor VLR deletes the user's profile and sends a cancellation acknowledgement to the old ILD.

8. and 10. The old ILD and the HLR send an acknowledgement message to the current ILD and update their tables. If distant pointers exist, then go to step 11, otherwise go to step 13 .

9. The current ILD sends an update message to all distant ILDs.

10. Distant ILDs update their pointer and send an acknowledgement to the current ILD.

11. The current ILD sends an acknowledgement message to the current MSC/VLR.

\subsubsection{Location search procedures}

The search procedure of a called mobile requires determining the current location area of this one. Figures 6 to 11 show the six various possible scenarios according to the strategy defined above.

Scenario 1: The first scenario (Fig. 6) presents the case where the called mobile and the calling mobile are in the same ILD and the called MUs is in its anchor location area.

1. A call is initiated to an MU and forwarded to the MSC/VLR of the calling unit.

2. The MSC sends a location request to its associated ILD.

3. The ILD determines the anchor LA of the called mobile and forwards the location request to the anchor MSC.

4. The anchor MSC assigns a TLDN to the called mobile and transmits it to the calling MSC.

5. The calling MSC sets up a connection with the called MSC using the TLDN.

Scenario 2: The second scenario (Fig. 7) presents the case where the called mobile unit and the calling mobile unit are in the same ILD, but the called mobile unit does not move into its anchor LA. In this case, a pointer must be crossed to reach the called mobile.

1. A call is initiated to an MU and is transmitted to the MSC/VLR of the calling mobile unit.

2. The MSC sends a location request to the ILD.

3. The ILD determines the anchor LA of the called mobile unit and forwards the request to the anchor MSC.

4. The request is then forwarded by the anchor MSC to the current LA of the called mobile unit by crossing a pointer.

5. The current MSC assigns a TLDN to the called mobile unit and transmits it to the calling MSC.

6. The calling MSC sets up a connection with the called mobile unit using the TLDN. 


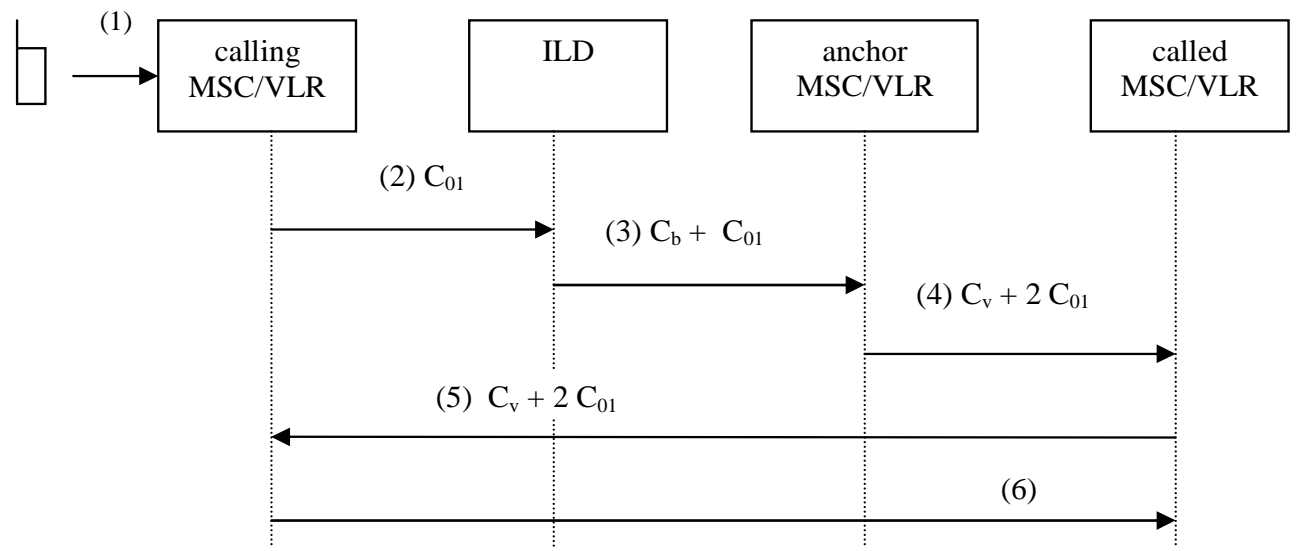

Fig. 7. Location search procedure (scenario 2).
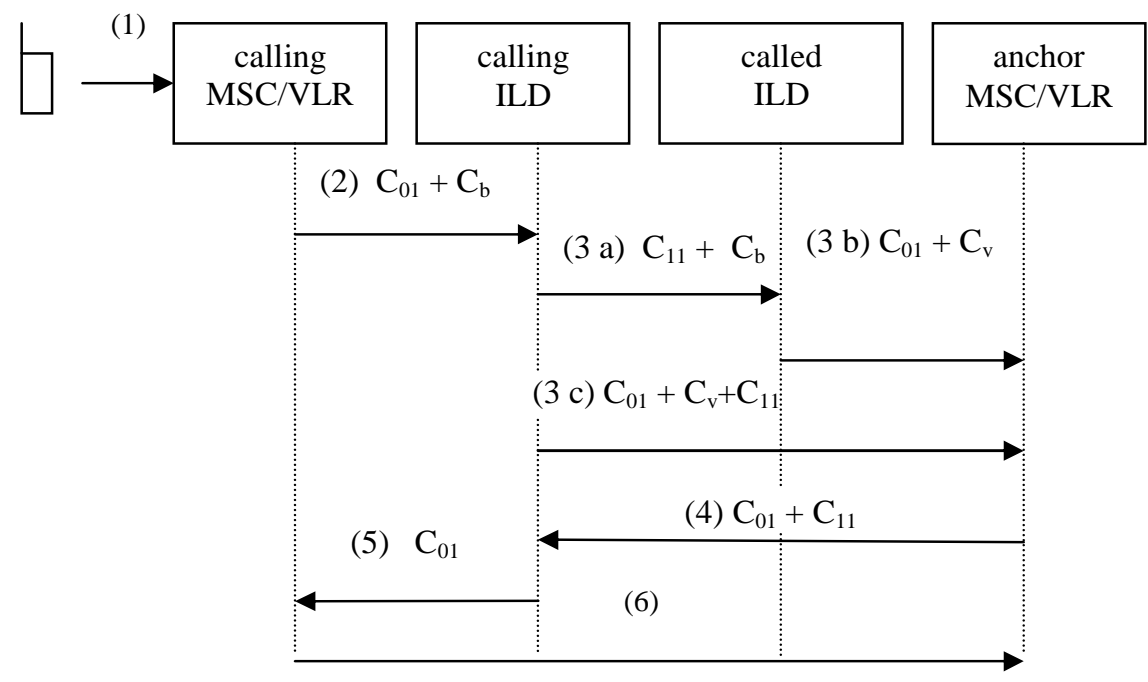

Fig. 8. Location search procedure (scenario 3).

Scenario 3: The third scenario (Fig. 8) presents the case where there is a distant pointer set up in the calling ILD and supposes that the called mobile unit moves into its anchor LA.

1. A call is initiated to an MU and is transmitted to the MSC/VLR of the calling mobile unit.

2. The MSC sends a location request to the calling ILD. If an indirect pointer is used, then:

3a) The ILD forwards the location request to the ILD of the called mobile unit.

3b) The called ILD forwards the message to the anchor LA of the called mobile unit.

If a direct pointer is used then:

3c) The ILD forwards the location request to the anchor LA of the called mobile unit.

3. The anchor MSC assigns a TLDN to the called mobile unit and transmits it to the calling ILD.

4. The calling ILD forwards the TLDN to the calling MSC.

5. The calling MSC sets up a connection to the called mobile unit using the TLDN. 


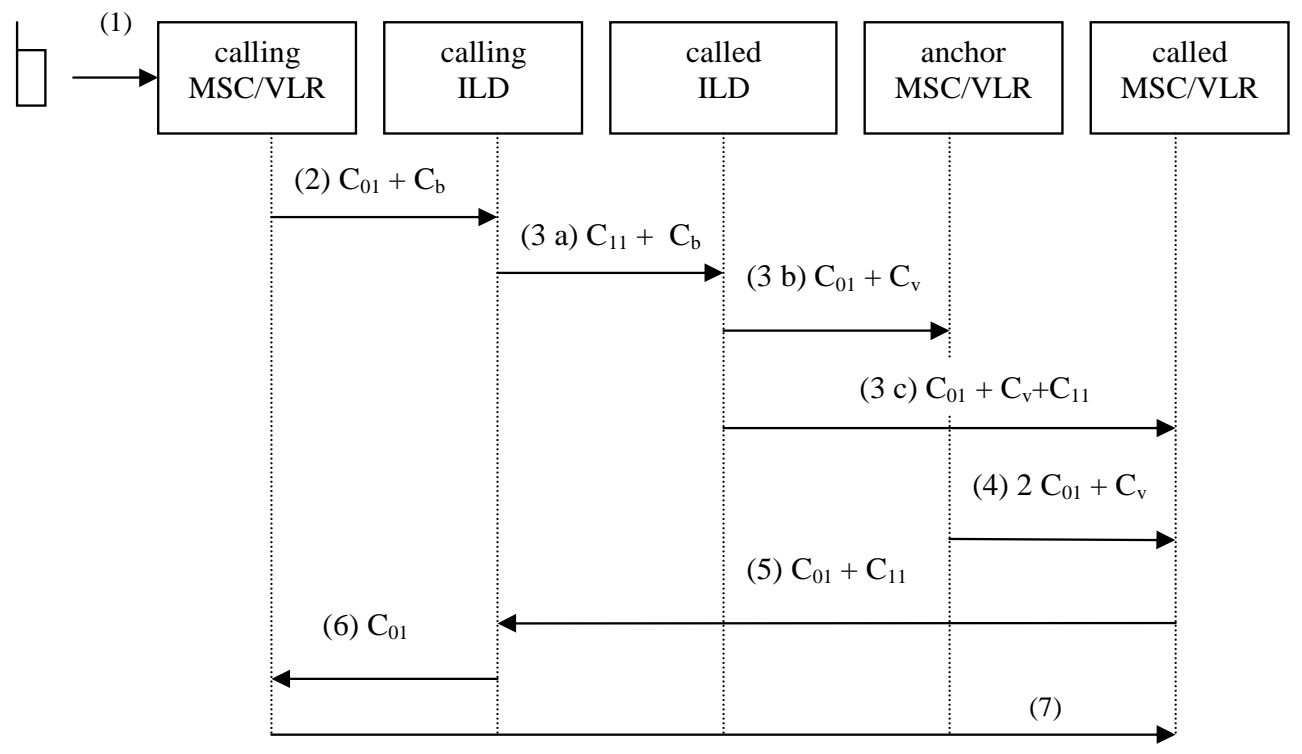

Fig. 9. Location search procedure (scenario 4).

Scenario 4: The fourth scenario (Fig. 9) presents the case where there is a distant pointer set up in the ILD of the calling mobile unit and the called mobile unit does not move into its anchor LA.

1. A call is initiated to an MU and is transmitted to the MSC/VLR of the calling mobile unit.

2. The MSC sends a location request to the calling ILD.

If an indirect pointer is used then:

3a) The ILD transmits the location request to the ILD of the called mobile unit.

$3 b)$ The called ILD forwards the message to the anchor location area of the called mobile unit.

If a direct pointer is used, then:

3c) The ILD transmits the location request to the anchor location area of the called mobile unit.

3. The request is then forwarded from the anchor MSC to the current LA of the called mobile unit by crossing a pointer.

4. The current MSC assigns a TLDN to the called mobile unit and transmits it to the calling ILD.

5. The calling ILD forwards the TLDN to the calling MSC.

6. The calling MSC sets up a connection to the called mobile unit using the TLDN.

Scenario 5: The fifth scenario (Fig. 10) presents the case where there is no data relative to the called mobile unit in the calling ILD and supposes that the called mobile unit moves into its anchor LA.

1. A call is initiated to an MU and is transmitted to the MSC/VLR of the calling mobile unit.

2. The MSC sends a location request to the calling ILD.

3. The ILD forwards the location request to the HLR of the called mobile unit.

4. The HLR determines the ILD of the called mobile unit as well as its anchor LA, and sends the request to the anchor LA.

5. The called ILD forwards the message to the anchor location area of the called mobile unit.

6. The anchor MSC assigns a TLDN to the called mobile unit and transmits it to the calling ILD. 


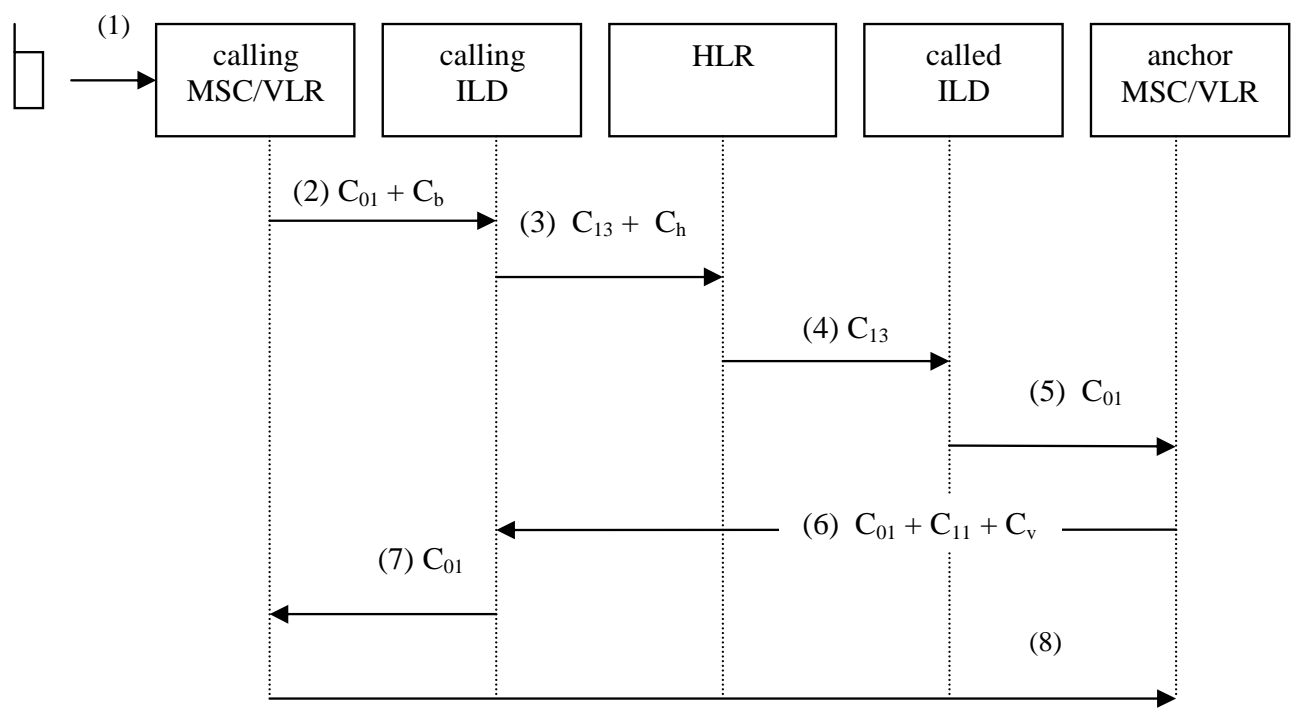

Fig. 10. Location search procedure (scenario 5).

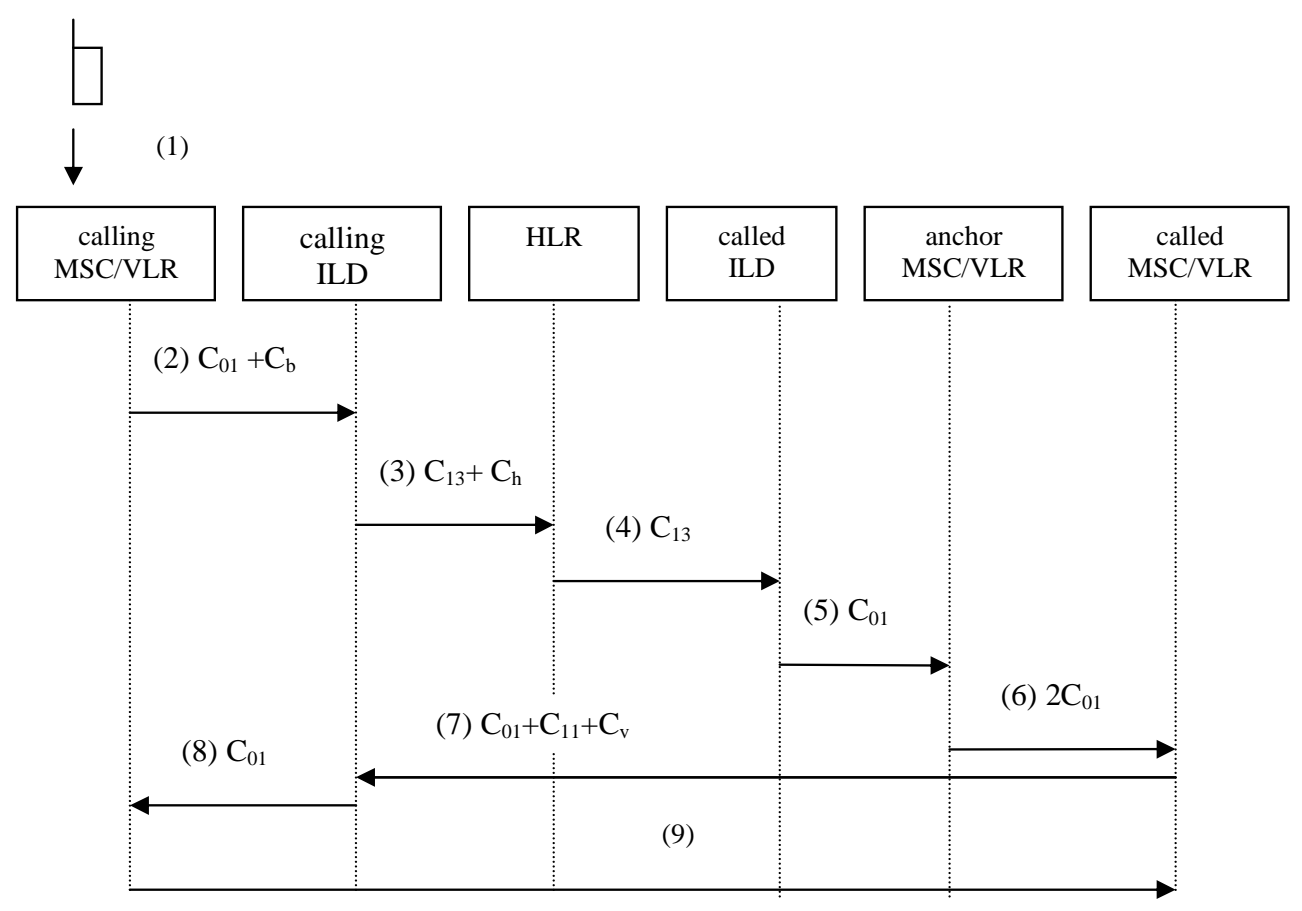

Fig. 11. Location search procedure (scenario 6).

7. The calling ILD forwards the TLDN to the calling MSC.

8. The calling MSC sets up a connection to the called mobile unit using the TLDN.

Scenario 6: The sixth scenario (Fig. 11) presents the case where there is no data relative to the called mobile unit in the calling ILD and the called mobile unit does not move into its anchor LA. 
1. A call is initiated to an MU and is transmitted to the MSC/VLR of the calling mobile unit.

2. The MSC sends a location request to the ILD.

3. The ILD forwards the location request to the HLR of the called mobile unit.

4. The HLR determines the ILD of the called mobile unit as well as its anchor LA, and sends the request to the ILD.

5. The called ILD forwards the message to the anchor location area of the called mobile unit.

6. The request is then forwarded from the anchor MSC to the current LA of the called mobile unit by crossing a pointer.

7. The current MSC assigns a TLDN to the called mobile unit and transmits it to the calling ILD.

8. The calling ILD forwards the TLDN to the calling MSC.

9. The calling MSC sets up a connection to the called mobile unit using the TLDN.

The main difference between the user location strategy employing forwarding pointers proposed in [6] and the proposed scheme reposed on the use of a new level of intermediate location database (ILD). This new architecture is built on a two levels distributed architecture both using pointers to track users instead of one pointer level as in [6]. The introduction of the ILDs to manage the location of the users in a region can be compare to the Mobile anchor Point (MAP) used in HMIPv6 [12] to reduce the signaling. There is also the semi-dynamic approach to setup the direct and indirect pointers to the users. The pointer setup is based on the classification of the users depending on their movement. The dynamic approach supposed the calculation for each location search of the cost of the procedure. This could lead to a huge processing load at the ILD level.

\section{Performance analysis}

In this section, we first present the analytical model of the proposed strategy and then the numerical results of the performance analysis by comparison with IS-41 standard.

\subsection{Analytical model}

The various procedures presented in the preceding section imply the exchange of several signalization messages among the network elements. The costs of signalization traffic and database loads depend, in general, on the MU location. These costs can be represented by the delays when establishing a connection during a location update or search procedure and are considered as the most significant criteria of performance. Indeed, according to the user, the quality of service of the network is intrinsically related to the connection delay.

In this model, we suppose that the link and the database access costs are defined on the delay basis respectively of a signalization message transmission and an update or a query of the databases. Moreover, we suppose that an MSC/VLR covers only one location area. For each mobile unit, we define the following quantities:

$\lambda$ : Average number of calls to a target MU per time unit;

$\mu$ : Average number of times the user changes LA per time unit;

$q$ : Probability that the new location area (MSC/VLR) after a move is in the same ILD as the previously visited location area;

$r$ : Probability that the called MU is found in its anchor LA;

$t$ : Probability that the calling mobile unit and the called mobile unit are in the same ILD; 
$v$ : Probability that a pointer exists towards a called mobile in the ILD.

For the various operations introduced in Section 3, we define the following costs:

$U_{\text {intra }}$ : Cost for a location update operation when the MU's move is intra-ILD;

$U_{\text {inter: }}$ : Cost for a location update operation when the MU's move is inter-ILD;

$S_{1}$ : Cost for a search procedure operation using scenario 1 , which means that the called mobile unit and the calling mobile unit are in the same ILD, and the called mobile unit roams in its anchor LA;

$S_{2}$ : Cost for a search procedure operation using scenario 2, which means that the called mobile unit and the calling mobile unit are in the same ILD, and the called mobile unit does not roam in its anchor LA;

$S_{3}$ : Cost for a search procedure operation using scenario 3, which means that there is a pointer in the ILD of the calling mobile unit towards the called mobile unit and the called mobile unit roams in its anchor LA;

$S_{4}$ : Cost for a search procedure operation using scenario 4 , which means that there is a pointer in the ILD of the calling mobile unit towards the called mobile unit and the called mobile unit does not roam in its anchor LA;

$S_{5}$ : Cost for a search procedure operation using scenario 5 , which means that there is no pointer in the calling ILD and the called mobile unit roams in its anchor LA;

$S_{6}$ : Cost for a search procedure operation using scenario 6 , which means that there is no pointer in the calling ILD and the called mobile unit does not roam in its anchor LA;

$U_{T}$ : Total cost for a location update procedure;

$S_{T}$ : Total cost for a location search procedure;

$C_{G}$ : Total cost for a location update and search procedures.

The total costs according to the quantities above are as follows:

$$
\begin{aligned}
& U_{T}=q U_{\text {intra }}+(1-q) U_{\text {inter }} \\
& \left.S_{T}=t\left(r S_{1}+(1-r) S_{2}\right)+(1-t)\left(v\left(r S_{3}+(1-r) S_{4}\right)+(1-v) r S_{5}+(1-r) S_{6}\right)\right) \\
& C_{G}=\mu U_{T}+\lambda S_{T}
\end{aligned}
$$

In order to determine the total costs defined above, it is necessary to define the link costs and the database access costs of the various network elements. To simplify the model, we suppose that the cost of a database update is equal to a database query. Moreover, we suppose that these costs are fixed and do not depend on day time or traffic load.

The various costs are the following:

$C_{01}$ : Cost of transmitting a message between SSP (MSC/VLR) and LSTP (ILD);

$C_{11}$ : Cost of transmitting a message between one LSTP (ILD) and another LSTP;

$C_{13}$ : Cost of transmitting a message between a LSTP (ILD) and SCP (HLR);

$C_{v}$ : Cost of a database update or query at the VLR;

$C_{b}$ : Cost of a database update or query at the ILD;

$C_{h}$ : Cost of a database update or query at the HLR. 
Table 1

Expression of the location search scenario costs

\begin{tabular}{ll}
\hline Scenario & Cost \\
\hline 1 & $4 C_{01}+C_{v}+C_{b}$ \\
2 & $6 C_{01}+2 C_{v}+C_{b}$ \\
$3:$ with distant pointer & $4 C_{01}+2 C_{11}+C_{v}+2 C_{b}$ \\
: with direct pointer & $4 C_{01}+2 C_{11}+C_{v}+C_{b}$ \\
$4:$ with distant pointer & $6 C_{01}+2 C_{11}+2 C_{v}+2 C_{b}$ \\
: with direct pointer & $6 C_{01}+2 C_{11}+2 C_{v}+C_{b}$ \\
5 & $4 C_{01}+C_{11}+2 C_{13}+C_{v}+C_{h}$ \\
6 & $6 C_{01}+C_{11}+2 C_{13}+2 C_{v}+C_{h}$ \\
\hline
\end{tabular}

In what follows, we suppose that, during a procedure, we access a database only once because the user's profile is loaded in the dynamic memory the first time we access the database and it is recorded in the database at the end of the procedure. Table 1 presents the various costs of the location search procedure.

Based on the location update operation shown in Fig. 4, the location update cost for an intra-ILD move is given by:

$$
U_{\text {intra }}=8 C_{01}+2 C_{v}
$$

Based on the location update operation shown in Fig. 5, the location update cost for an inter-ILD move is given by:

$$
U_{\text {inter }}=\left(8 C_{01}+2 C_{11}+2 C_{13}\right)+2 C_{v}+2 C_{b}+C_{h}+\sum_{k} U_{k}
$$

where $U_{k}=2 C_{11}+C_{b}$ and $k$ denotes the number of pointers to update.

According to the expressions Eqs (4) and (5), we can derive the total cost of the location update procedure

$$
U_{T}=q U_{\text {intra }}+(1-q) U_{\text {inter }}
$$

According to Table 1, we can derive the total cost of the location search procedure:

$$
\left.S_{T}=t\left(r S_{1}+(1-r) S_{2}\right)+(1-t)\left(v\left(r S_{3}\right)+(1-r) S_{4}\right)+(1-v)\left(r S_{5}+(1-r) S_{6}\right)\right)
$$

In order to compare the proposed strategy with that used in IS-41standard, it is necessary to determine the IS-41 cost of the location update and search procedures. According to Figs 1 and 2:

$$
\begin{aligned}
& U_{\text {is } 41}=4 C_{01}+4 C_{13}+C_{v}+C_{g}+C_{h} \\
& S_{\text {is } 41}=6 C_{01}+C_{11}+4 C_{13}+C_{v}+C_{g}+C_{h}
\end{aligned}
$$

The total cost for an update and search procedure is:

$$
C_{\text {is } 41}=\mu U_{\text {is } 41}+\lambda S_{\text {is } 41}
$$

In order to compare the proposed strategy with IS-41 standard, we define the relative cost as follows:

$$
\frac{C_{G}}{C i s 41}=\frac{U t+C M R * S t}{U_{\text {is } 41}+C M R * S_{\text {is } 41}}
$$

where $\mathrm{CMR}=\lambda / \mu$ is the ratio of the number of calls per unit of times to the number of changes of location area per unit of time for a given mobile unit. 


\subsection{Numerical results}

In this section, we present the numerical results of the proposed model. We will first define the different parameters of the numerical analysis and then analyze the results in comparison with those of IS-41 standard.

\subsubsection{Experiment parameters}

In the model hereafter, we suppose that an ILD consists of $x^{*} x$ LAs arranged in square. Moreover, MUs are assumed to be uniformly distributed in all the ILD area and have a constant speed in all directions with equal probability. We also assume that each MU exhibits the same arrival call rate at every MSC/VLR. Furthermore, each time an MU leaves an LA, one of the four sides of the square is crossed with equal probability. We also assume that all SSPs of the network is uniformly distributed among LSTPs and that each SSP corresponds to only one location area. We consider the case of the American PSTN where there is 160 LATA (Local Access Transport Area) through 7 areas RBOC (Regional Bell Operating Company). We suppose that a LATA represents only one LSTP. Each LATA is made up of 1250 SSPs. We deduce that there is on average 23 LSTPs per area. We suppose that the proposed model covers one area. It thus consists of 23 ILDs because those are located on the level of LSTPs. We can then dedyce the probability q that the new location area after a move is in the same ILD as that the previously visited the probability $r$ that a called mobile unit is in its anchor LA, and the probability $t$ that the calling mobile unit and the called mobile unit are in the same ILD. The probability that an MU lies in a border LA of the ILD:

$$
P_{b}=\frac{4 *(x-1)}{x^{*} x}
$$

where $x$ denotes the length of the side in terms of location area of the ILD.

The probability that the MU moves isnter-ILD is thus:

$$
P_{i}=\frac{(x-1)}{x^{*} x}
$$

Hence

$$
q=1-\frac{(x-1)}{x^{*} x}
$$

However, $x=\frac{1250}{23}$. Hence $q=0.88$ is the probability that the new location area after a move is in the same ILD that the previously visited. Given that there are 23 ILDs, the probability that the calling mobile unit and the called mobile unit are in the same ILD is equal to $t=1 / 23$. Given that there are 64 location areas in an ILD, the probability that an MU is in its anchor LA is $r=1 / 64$. The probability that an existing pointer goes towards a called mobile unit in the ILD of the calling mobile unit, $v$, and the number of ILDs having a pointer, $K$, depend on the mobility parameters of the MU. The link's cost parameters are defined in Table 2.

Since the ILD is located near its associated MSC/VLR, the signalization messages exchanged between the ILD and one of the MSC/VLR under its area do not cross an LSTP. It results that the cost between level 0 and level 1 (Fig. 3) has the smallest value. All the other costs are normalized to C01. The group of the selected values allows studying the variation effect of parameters $\mathrm{C} 11$ and $\mathrm{C} 13$ on the performance of the proposed model. The parameters of access costs at the databases are defined in Table 3. 
Table 2

Link cost parameters

\begin{tabular}{ccrr}
\hline Series & $C_{01}$ & $C_{11}$ & $C_{13}$ \\
\hline 1 & 1 & 5 & 2 \\
2 & 1 & 5 & 10 \\
3 & 1 & 10 & 2 \\
4 & 1 & 10 & 10 \\
\hline
\end{tabular}

Table 3

Database access cost parameters

\begin{tabular}{cccr}
\hline Series & $C_{v}$ & $C_{b}$ & $C_{h}$ \\
\hline 5 & 3 & 1 & 6 \\
6 & 3 & 1 & 12 \\
7 & 6 & 1 & 6 \\
8 & 6 & 1 & 12 \\
\hline
\end{tabular}

Since an ILD access implies a simple lookup, the ILD access cost has the smallest value. All the other costs are normalized to $C_{b}$. The group of the selected values allows studying the variation effect of parameters $C_{v}$ and $C_{h}$ on the performance of the proposed model. We assume that the cost of procedure GTT, $C_{g}$, is $50 \%$ of that of the ILD access.

\subsubsection{Results analysis}

In this section, we analyze the numerical results of the comparison between IS-41 standard and the proposed model. The results are represented in graphic form according to the various parameters stated previously. To evaluate the performances of the proposed model, we carried out several numerical calculations for various values of the parameters quoted above. Moreover to simplify the analysis, we consider that either the link cost parameters or the database access costs dominate. Thus, when the link costs dominate, the access costs at the databases are nil and vice versa. The probability parameters used for end of calculations are $q=0.8, t=0.05, v=0.8, k=2$ and $r=0.01$.

Figure 12 represents the relative cost of the proposed model according to the CMR when link costs dominate. We note that the proposed model clearly improves the performances of IS-41 standard for the sets $C_{01} C_{11} C_{13}=1510$ and $C_{01} C_{11} C_{13}=11010$. For the sets $C_{01} C_{11} C_{13}=152$ and $C_{01} C_{11}$ $C_{13}=1102$, the two curves are similar and degrade IS-41 standard for values of CMR higher than one. This is explained by the fact that the proposed strategy implies the same procedures when the link cost $\mathrm{C} 11$ is higher than the link $\operatorname{cost} C_{13}$. It should be noted that this case is very rare because the cost of link towards the HLR is generally higher than that between two ILDs.

All in all, the proposed model improves IS-41 of almost 60\%. Moreover, we note that, when the CMR increases, the cost savings of the proposed model tend to decrease. It is necessary to stress that, when the CMR is small, the location update dominates while when CMR is large, the location search dominates. When the CMR increases, the relative cost increases because location search prevails and the fraction of cost avoided by location searches is smaller than that avoided by location updates.

Figure 13 represents the relative cost of the proposed model according to the CMR when database access costs dominate. We note that only the database access costs $C_{v} C_{b} C_{h}=616$ degrades the performances of IS-41 standard. In all the other cases, the relative cost is less. The results obtained for the group of parameters $C_{v} C_{b} C_{h}=616$ are explained by the fact that the proposed strategy avoids going up on the level of the HLR. Indeed, the updates take place in major part in the ILD. So access with the VLR is as expensive as access to the HLR. The access costs of the HLR and the VLR being equal, 


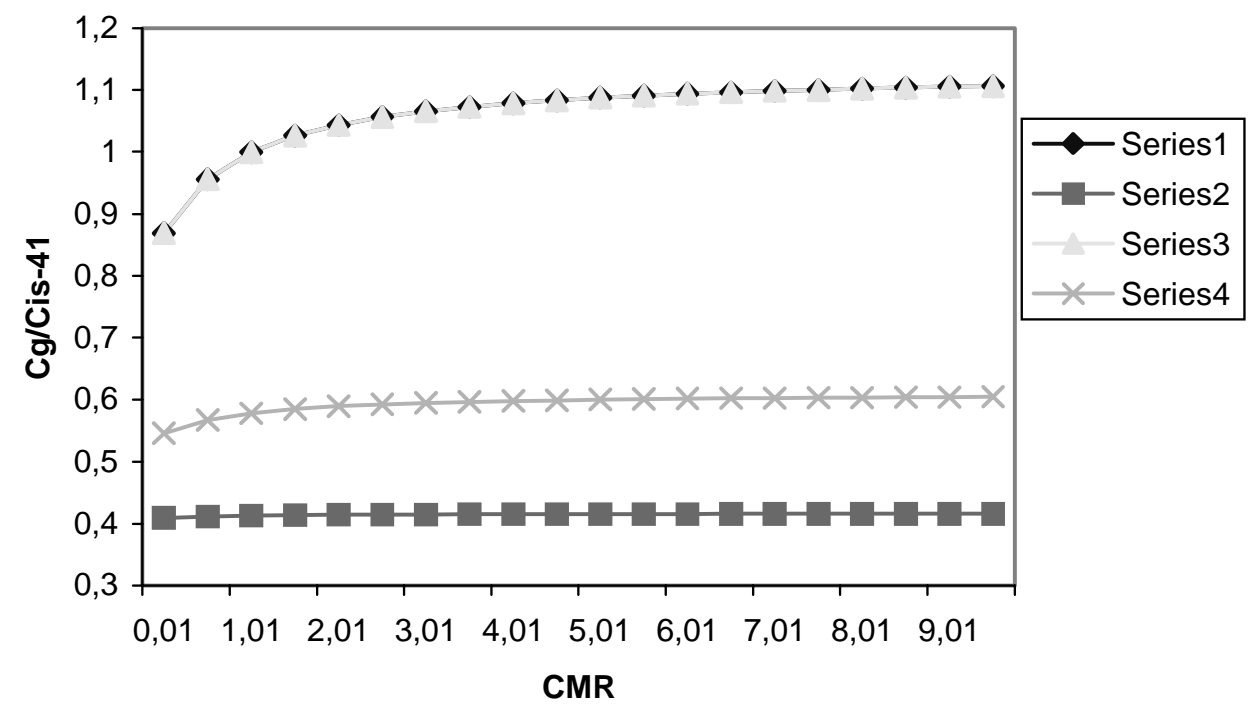

Fig. 12. Relative cost for the network's link parameters.

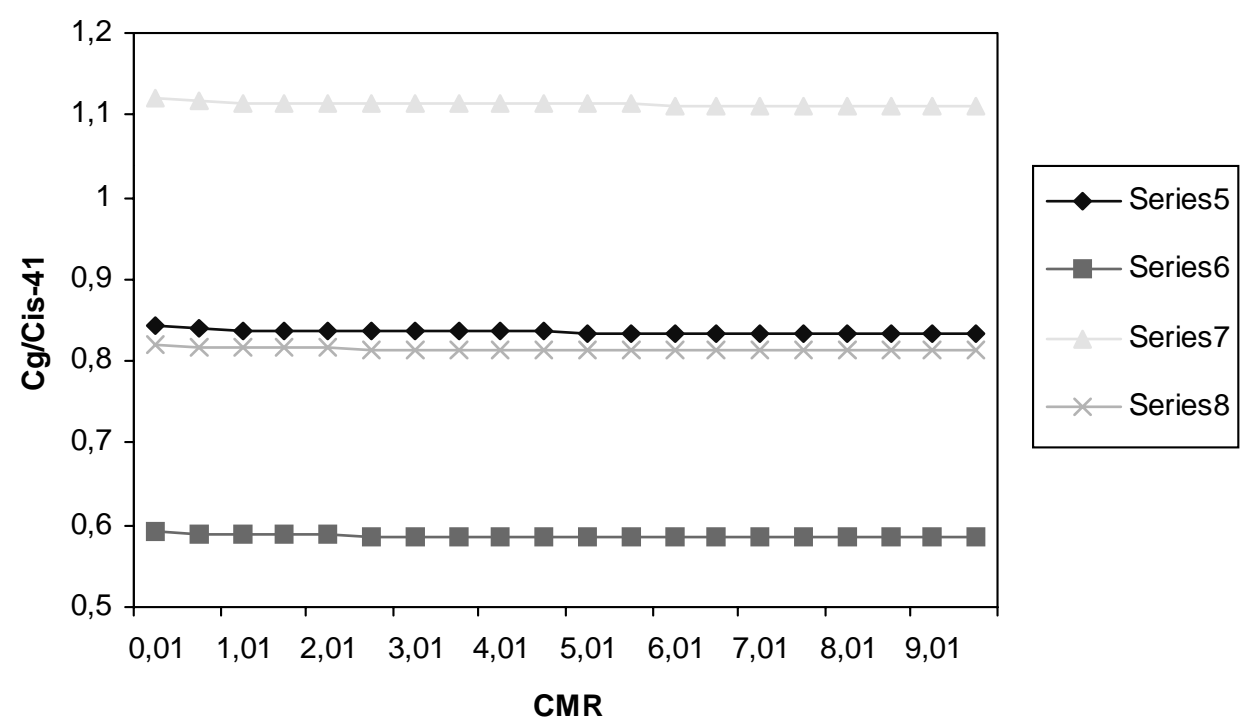

Fig. 13. Relative cost for the network's database access parameters.

the IS-41 standard is more powerful because the proposed model adds an ILD access. For all the other cases, the relative cost is less because the access cost to the HLR is larger than the access cost to the VLR. In this case, the proposed strategy improves IS- 41 of almost $40 \%$. The comparison of the sets of parameters 1, 2, 3 and 4 demonstrates that increase of the access cost of the VLR, $C_{v}$, implies a higher relative cost whatever the value of the CMR.

Figures 14 and 15 represent the relative cost of the proposed model according to the CMR when q varies. The sets of parameters are respectively: $C_{01} C_{11} C_{13}=1510$ and $C_{v} C_{b} C_{h}=316$. These two sets were selected because they represent the most probable case. These results show the variation effect of the user's mobility on the performances of the proposed algorithm. We note that when the probability 


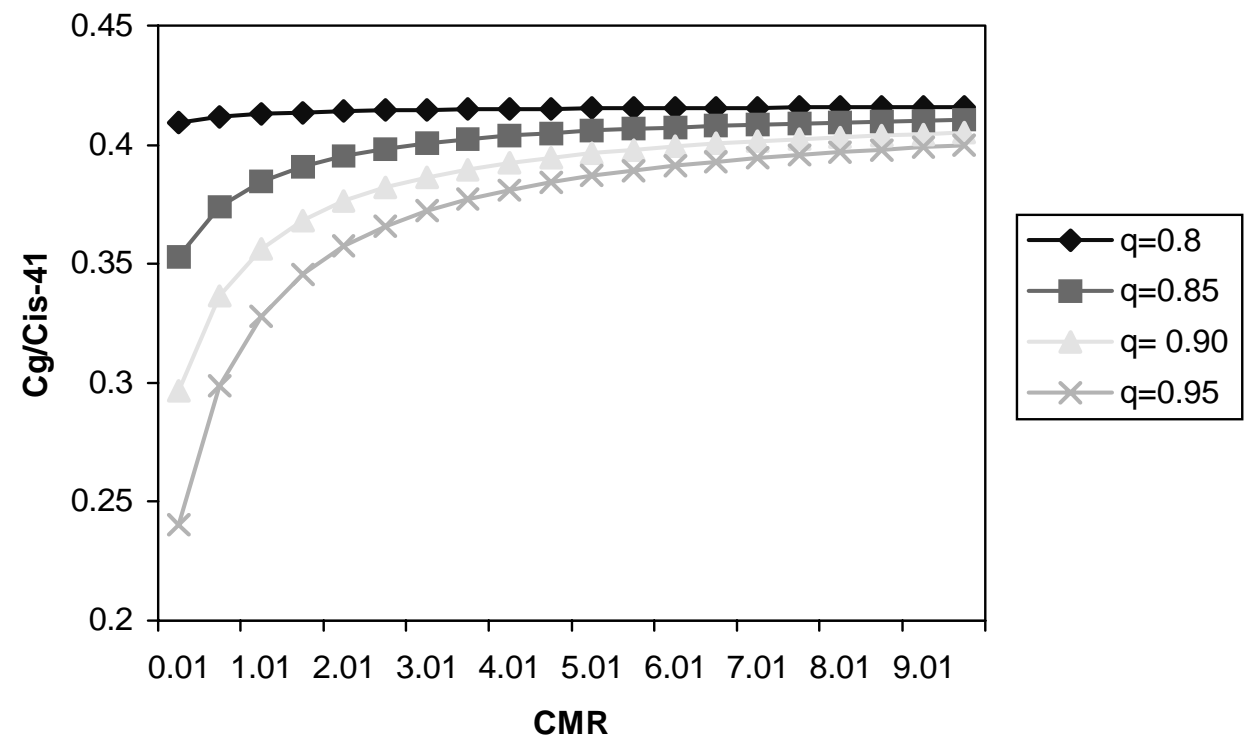

Fig. 14. Relative cost for the network's link parameters for different $q$ values.

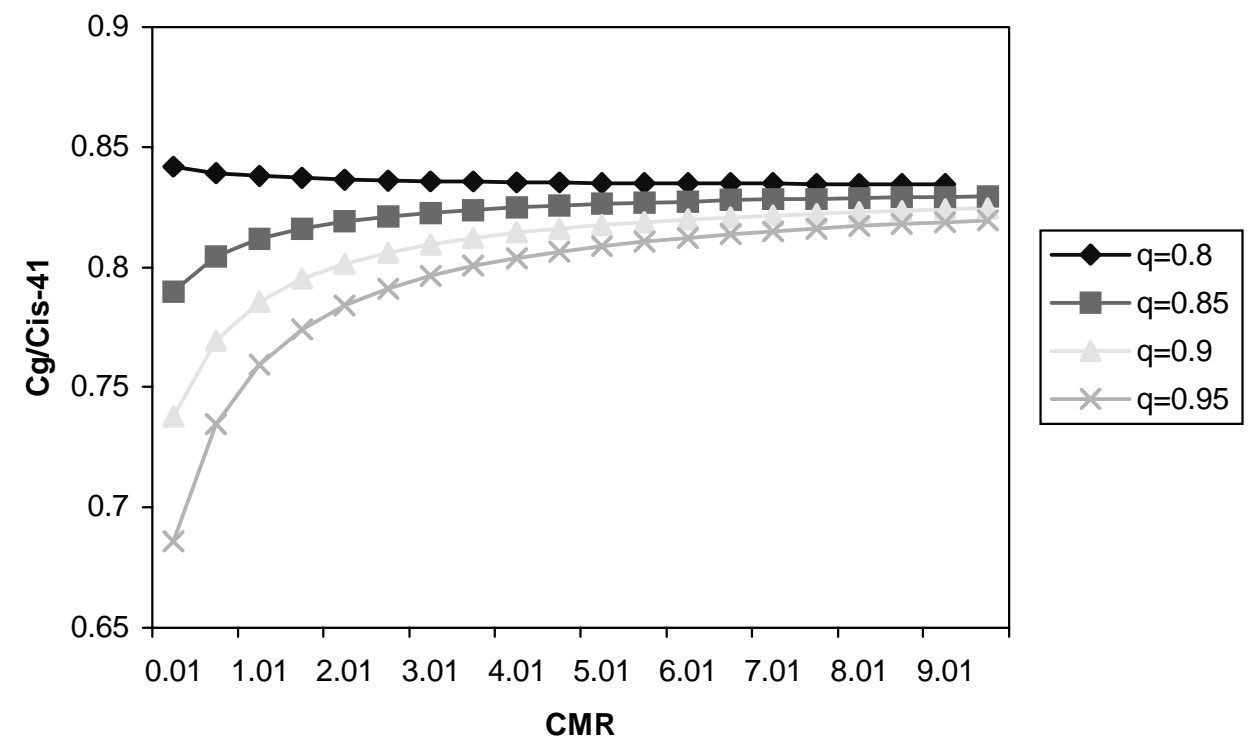

Fig. 15. Relative cost for the network's database access parameters for different $q$ values.

of intra-ILD moves is high, there is less requests towards the HLR and less updates of pointers, thus a less relative cost. In the opposite case, when $q$ decreases, the mobile often moving from an ILD to another, the requests towards the HLR and the updates of the pointers are much more frequent, resulting in a higher relative cost. For high values of the CMR, the frequency of moves inter-ILD is small even for small values of $q$, hence the convergence of the curves.

However, for small values of the CMR, the frequency of inter-ILD moves is high. Hence the diminution of the relative cost when q increases. 


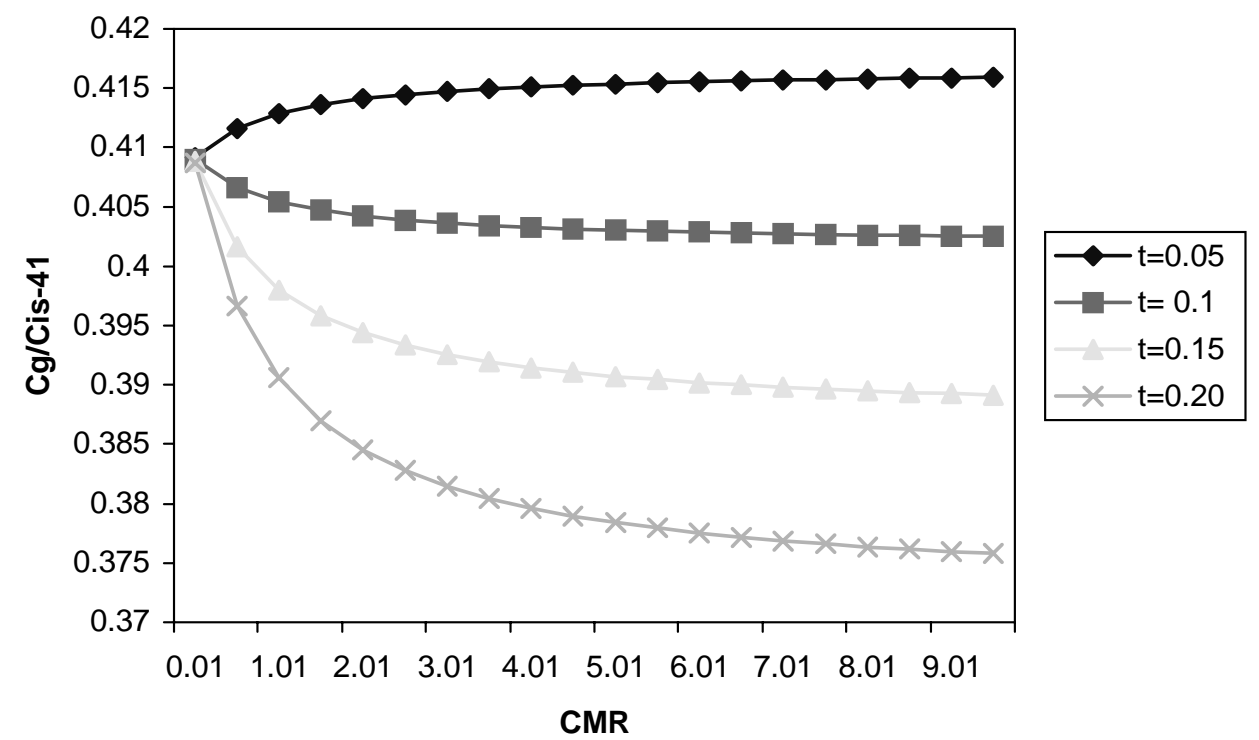

Fig. 16. Relative cost for the network's link parameters for different $t$ values.

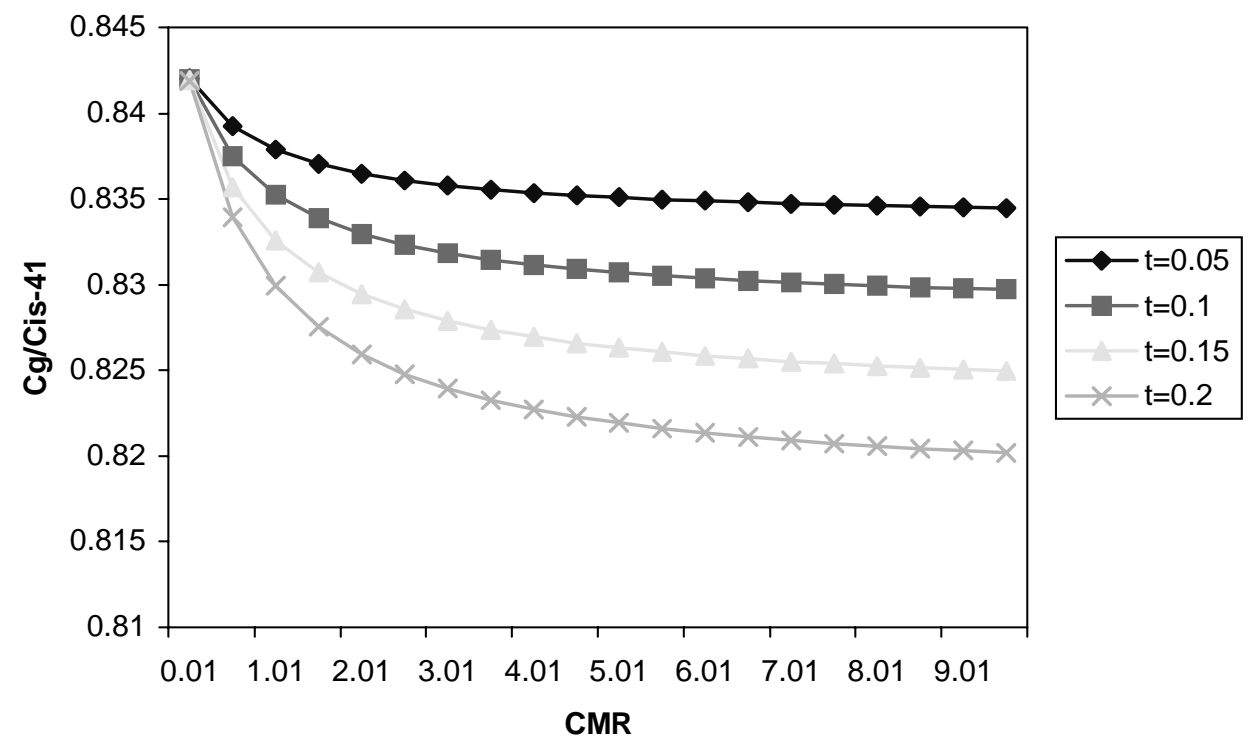

Fig. 17. Relative cost for the network's link parameters.

Figure 16 represents the relative cost of the proposed model according to the CMR when $\mathrm{t}$ varies. The group of parameters is: $C_{01} C_{11} C_{13}=1510$. We note that the relative cost decreases when $\mathrm{t}$ increases. This is due to the fact that the proposed strategy does not go up on the level of the HLR because the calling mobile unit and the called mobile unit are in the same ILD. So for small values of CMR, the relative cost almost does not vary according to $t$ because the updates dominate (Eq. (3)). However, for great values of CMR, the relative cost is less when $t$ increases because the search procedure dominates.

Figure 17 represents the relative cost of the proposed model according to the CMR when $t$ varies. The 


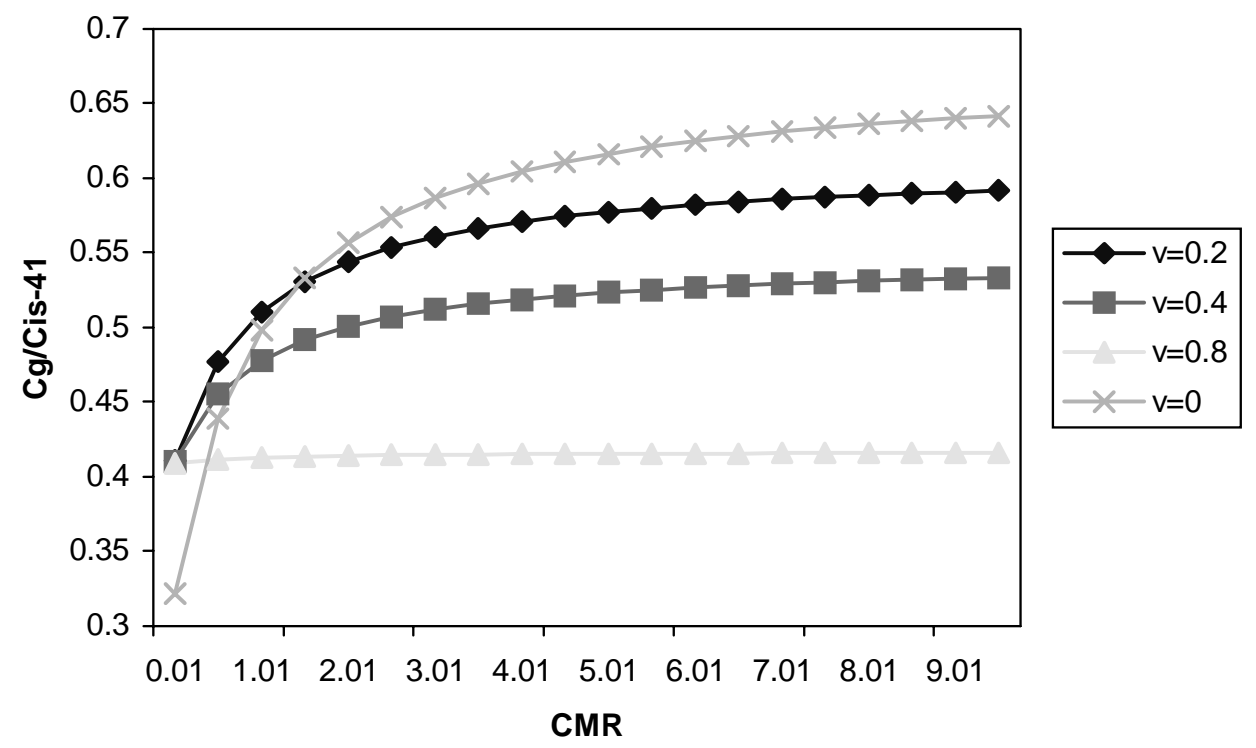

Fig. 18. Relative cost for the network's link parameters.

group of parameters is: $C_{v} C_{b} C_{h}=316$. We note that the relative cost decreases when $\mathrm{t}$ increases. This is due to the fact that the proposed strategy does not go up on the level of the HLR because the calling mobile unit and the called mobile unit are in the same ILD. So for small values of CMR, the relative cost almost does not vary according to t because the updates dominate (Eq. (3)). However, for high values of CMR, the relative cost is less when $t$ increases because the search procedure dominates.

Figures 18 and 19 represent the relative cost of the proposed model according to the CMR when $\mathrm{v}$ varies. The sets of parameters are respectively: $C_{01} C_{11} C_{13}=1510$ and $C_{v} C_{b} C_{h}=316$. We note that, for small values of CMR, the relative cost is less when $v$ are small while, for great values of CMR, the relative cost increases to a significant degree.

This is explained by the fact that when $v$ is small, there are less pointers thus less updates of the pointers. However, for the search procedure, the proposed strategy sends requests to the HLR, hence a higher cost when the CMR increases. The comparison of the curves $v=0$ and $v=0.8$ shows that the fact to have pointers improves the performances of the proposed model when the CMR increases.

The performance study shows that the proposed architecture can reduce the total cost of location management by $75 \%$. This reduction is significant compare to the $60 \%$ of the user location strategy employing forwarding pointers defines in [6]. The introduction of the ILDs reduce the total cost by $15 \%$.

\section{Concluding remarks}

In this paper, we proposed a method to improve the update and search procedures in mobility management, generally based on IS-41 standard, by taking into account the specific characteristics of the traffic in the mobile networks. Indeed, we proposed an algorithmic model to update the location databases of mobile units and to locate these mobiles by minimizing the location update and search costs, while reducing the number of access to the nominal database of the network, and consequently the load of traffic generated by the mobility management procedures. The proposed architecture combines to some extent two architectures, centralized and distributed, on which recent searches in location management 


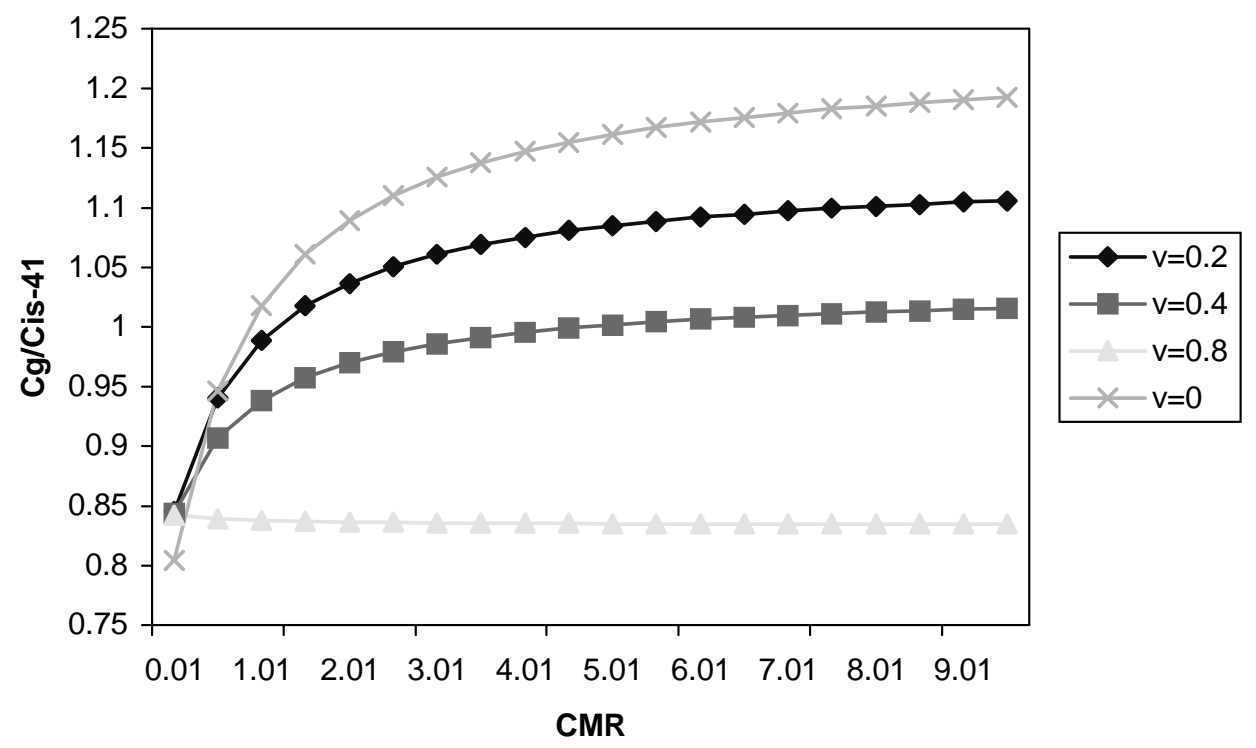

Fig. 19. Relative cost for the network's database access parameters.

are based. This hybrid architecture eliminates the majority of disadvantages of centralized architecture and distributed architecture and introduced a semi-dynamic approach without the load related with the dynamic models. In this model, we also improved and integrated the local strategy of databases per user, which consists of allocating to each user an anchor database to which is deferred all the updates carried out in the same ILD. This allowed to improve in a significant way the location procedure's efficiency of the mobile unit and thus to apply the proposed model to various user classes dependently on the CMR.

\section{References}

[1] EIA/TIA., Interim Standard IS-41-B, Cellular Radio-Telecommunications Intersystem Operations, December 1991.

[2] EIA/TIA., Interim Standard IS-41-C, Cellular Radio-Telecommunications Intersystem Operations, February 1996.

[3] G.M. Choi and D.H, Cho, Performance Analysis of Cache Strategy for Signaling Traffic Management in a Wireless ATM Network, Wireless Networks (6) (2000), 355-362.

[4] J.S.M. Ho and I.F. Akyildiz, Dynamic Hierarchical Database Architecture for Location Management in PCS Networks, IEEE/ACM Transactions on Networking 5(5) (October, 1997), 646-660.

[5] J. Ho and I.F. Akyildiz, Mobile User Location Update and Paging Under Delay Constraint, Wireless Networks 1(4) (1995), 413-425.

[6] R. Jain and Y.B. Lin, An Auxiliary User Location Strategy Employing Forwarding Pointers to Reduce Network Impacts of PCS, ACM-Baltzer J. Wireless Networks 1(2) (1995), 197-210.

[7] R. Jain, Y.B. Lin, C. Lo and S. Mohan, A Caching Strategy to Reduce Network Impacts of PCS, IEEE Journal on Selected Areas in Communications 12(8) (October, 1994), 1434-1444.

[8] H. Safa, S. Pierre and J. Conan, An Efficient Location Management Scheme for PCS Networks, Computer Communications 24(14) (August, 2001), 1355-1369.

[9] S. Tabbane, Location Management Methods for 3rd Generation Mobile Systems, IEEE Communications Magazine 35(8) (August, 1997), 72-78.

[10] W. Ma and Y. Fang, Two-Level Pointer Forwarding Strategy for Location Management in PCS Networks, IEEE Transactions on Mobile Computing 1(1) (January, 2002), 32-45.

[11] W. Wang and I.F. Akyildiz, A New Protocol for Intersystem Roaming in Next-Generation Wireless System, IEEE Journal on Selected Areas In Communications 19(10) (October 2001).

[12] H. Soliman et al., Hierarchical MIPv6 mobility management HMIPv6, Internet Draft, draft-ietf-mobileip-hmipv6-06.txt, July, 2002. 
Charles Abondo received an M.Sc., degree in physics from the University of Limoges in 1998, a bachelor degree in computer engineering from Ecole Polytechnique of Montreal in 2000 and an M.Sc.A. in computer engineering in 2001 from the same institution. He is currently completing his Ph.D. at Ecole Polytechnique of Montreal. His research interests include mobility and QoS problems in next generation systems.

Samuel Pierre received the B. Eng, degree in civil engineering in 1981 from École Polytechnique de Montréal, Québec, the B. Sc. and M. Sc. degrees in mathematics and computer science in 1984 and 1985, respectively, from the UQAM, Montréal, the M. Sc. degree in economics in 1987 from the Université de Montréal, and the Ph.D. degree in Electrical Engineering in 1991 from École Polytechnique de Montréal. Dr. Pierre is currently a Professor of Computer Engineering at École Polytechnique de Montréal where he is Director of the Mobile Computing and Networking Research Laboratory (LARIM) and NSERC/Ericsson Industrial Research Chair in Next-generation Mobile Networking Systems. He is the author of four books, co-author of two books and seven book chapters, as well as over 250 other technical publications including journal and proceedings papers. His research interests include wireline and wireless networks, mobile computing, performance evaluation, artificial intelligence, and electronic, learning. He is a Fellow of Engineering Institute of Canada, senior member of IEEE, a member of ACM and IEEE Communications Society. He is an Associate Editor of IEEE Communications Letters and IEEE Canadian Review, and he serves on the editorial board of Telematics and Informatics published by Elsevier Science. 

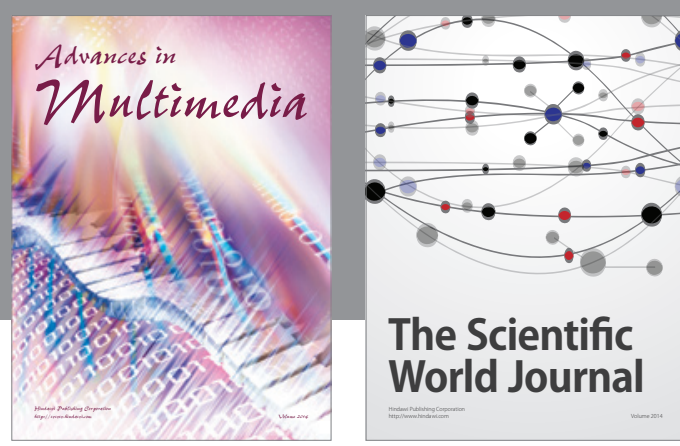

The Scientific World Journal
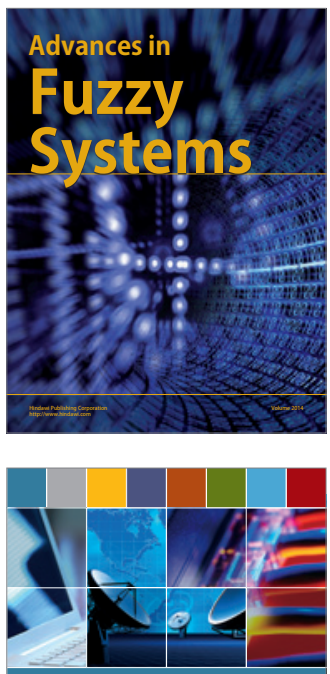

Computer Networks and Communications
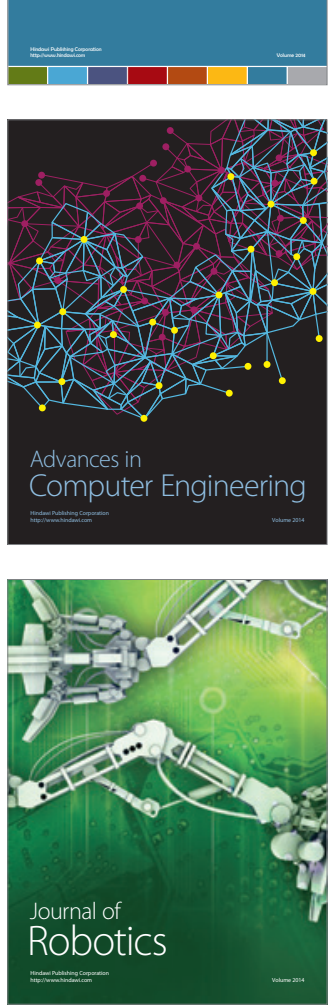
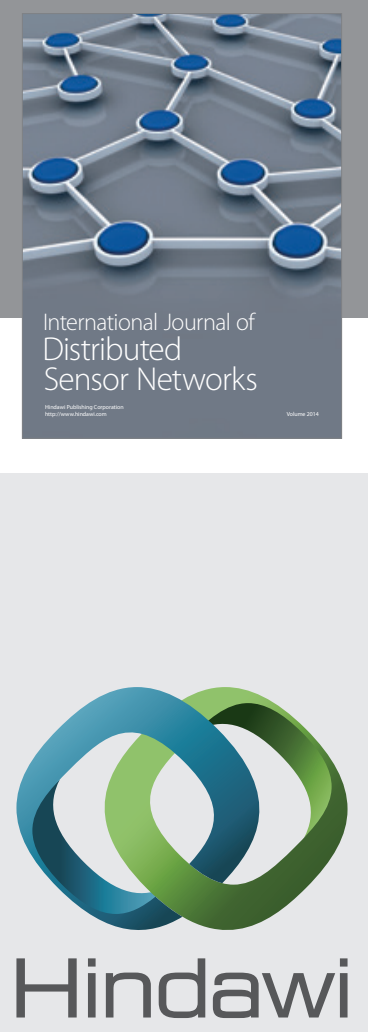

Submit your manuscripts at

http://www.hindawi.com
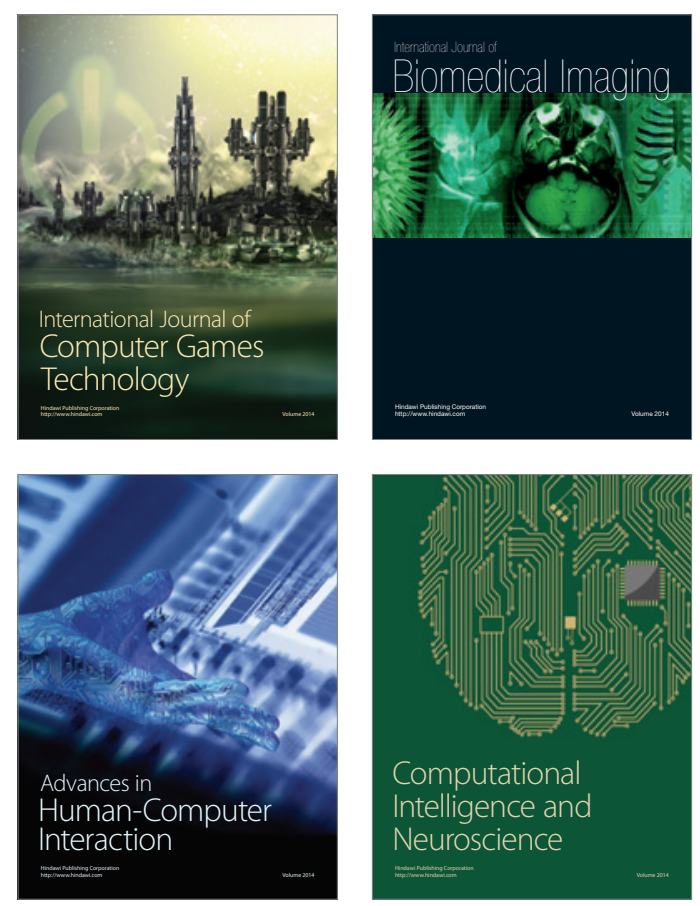
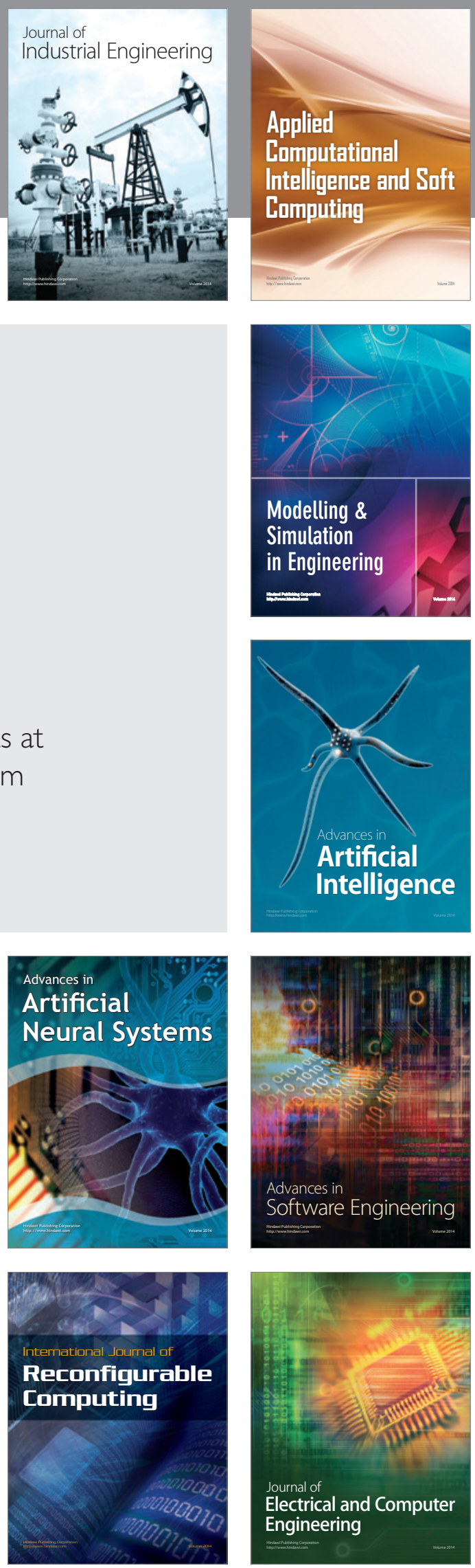